\title{
Evaluation of the aerothermodynamic field produced by a pseudospheric body of mercury type at $M=22.6$ flying in air in thermodynamic equilibrium (*)
}

\author{
D. Cunsolo - S. Angelucci
}

Riceruto il 19 Febbraio 1963

\begin{abstract}
SuMARY. - The differential equations valid after the shock are first given in curvilinear coordinates; the chosen unknowns are the two velocity components and the entropy and enthalpy. A function of entrops and enthalpy is then determined, by means of which all the thermodynamic variables of the fluir are "coherently" approximated. Later on, the density and all the other kinematic and thermodynamic variables are calculated immediately after the shock, taking the angle $\sigma$ as a parameter. The shape of the body is now taken into account and a convenient shape of the shock wave is given.

The differential equations are then integrated with a step-by-step procedure, until the stagnation entropy is reached on the body.

Finally the pressure and the temperature on the body are given. A sonic-to-stagnation pressure of 0.6 is the result, instead of 0.523 for a perfect gas.
\end{abstract}

Rrassusto. .... Somo prima ricavate dalla teoria dello strato d'urto sottile. le equazioni valide dietro l'onda d'urto prodotta da un corpo non appuntito viaggiante a velocità ipersoniea in atmosfera parzialmente dissociata che si suppone in equilibrio termodinamico. Come esempio si è considerata la capsula Mereury nella fase di rientro alla quota di $60 \mathrm{Km}$ ed alla velocità di $7500 \mathrm{~m} / \mathrm{sec}$. $\left(M_{\infty}=22,6\right)$ con la parte frontale in prossiniti dell'asse, completamente sferica.

Prima di iniziare lo studio del campo aerotermodinamieo dietro londa d'urto con l'" Inverse Problem ", sono determinati i coeficienti di una funzione dell'entropia ed entalpia da cui dipendono tutte le grandezze interessanti il fluido, funzione che tiene conto della dissociazione del gas.

1)i poi viene calcolata la densità nei punti immediatamente dietro l'onda per varii valori dell'angolo che la tangente alla stessa forma con las-

(*) Nota presentata al 20 Congresso Internazionalo Tecnico Scientifico dello spazio. Roma. 19-23 Giugno 1962. 
se del corpo, adoperando un metodo di approssimazioni successive, senza assegrnare ancora la forma dell'onda d'urto.

Dal calcolo delle densità sono note velocità, entropia ed entalpia dietro l'urto. Assegnando ora un'onda probabile si inizia il calcolo del campo dietro l'urto con le equazioni in forma adimensionale mediante un procedimento al passo, fino a giungere alla forma del corpo corrispondente all'onda d'urto data.

L'ultimo passo del procedimento di calcolo fornisce temperature e pressioni sul corpo. Calcolato così il campo subsonico dietro l'onda d'urto fino ed oltre la linea sonica, il eampo supersonico potrà, da questo punto, essere studiato col metodo delle caratteristiche.

Nymbols

$$
\begin{aligned}
& 1 \text { = Pressure } \\
& p_{o}=\text { Reference pressure (atmospheric pressure at sea level) } \\
& 0 \quad=\text { Density } \\
& T=\text { Temperature in oK } \\
& T_{o}=\text { Reference temperature }=273.16^{\circ} \mathrm{K} \\
& \varrho_{o} \quad=\text { Reference density }=p_{o} / R_{o} T_{o} \\
& R_{o}=\text { Gas constant referred to one } \mathrm{Kg} \text {. of air }=6.886 \times 10^{-2} \frac{\mathrm{Feal}}{\mathrm{K} \mathrm{g}^{\circ} \mathrm{K}} \\
& R=\text { Gas constant referred to one Kigr.mole } \\
& h \quad=\text { Enthalpy } \\
& \text { is = Entropy } \\
& F^{\prime}=p / p_{o} \\
& \text { i }=g / g_{0} \\
& \theta \quad=T / T_{0} \\
& \eta \quad=\frac{h}{R_{0} T_{o}}-300 \\
& \xi \quad=\frac{S}{R_{0}}-4 x \\
& \sigma \quad=\text { Shock wave angle } \\
& \sigma^{\prime} \quad=\frac{\pi}{2}-\sigma \\
& x \quad=\text { Curvilinear coordinate parallel to the shock wave } \\
& y=\text { Coordinate orthogonal to the shock wave } \\
& r ; z=\text { Cylindrical coordinates } \\
& K(x) \text { or } K\left(\sigma^{\prime}\right)=\text { Local curvature of the shock wave }(y=0) \\
& H \quad=1-K y \text { Correction factor for the curvature of the other coor- } \\
& \text { dinate lines }(y=\text { const.) }
\end{aligned}
$$




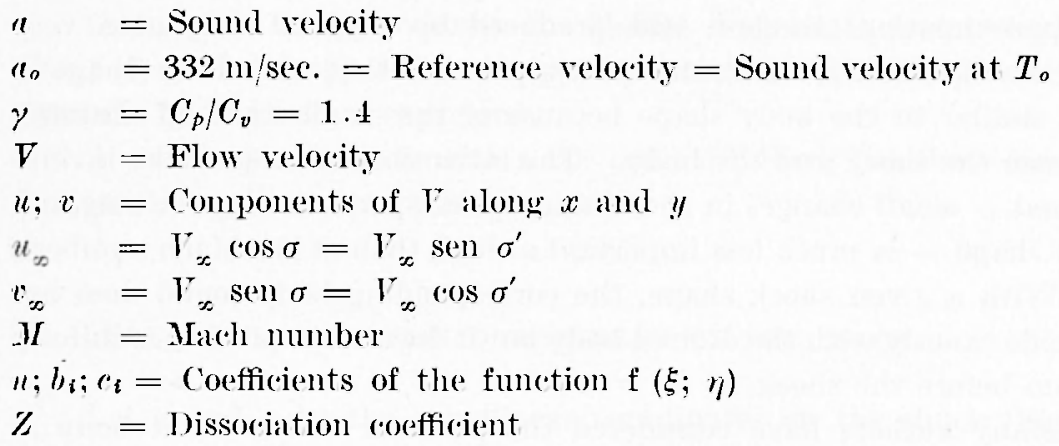

\section{Indexes}

$\therefore \quad=$ Reference ronditions

$\infty \quad=$ Free strean ronditions

$s \quad=$ Stagnation conditions

$(o r u)_{x}=\frac{\partial(o r u)}{\partial x} ; \quad 0 . x=\frac{\partial Q}{\partial x} ; \quad F_{\xi}=\frac{\partial F}{\partial \xi}$

$\left(\frac{\partial F}{\partial \eta}\right)_{\xi}=$ Derivative of $F$ with respect to $\eta$ at constant $\xi$.

\section{I - INTRomLCTION.}

The hypersonic flow introduces new problems in arodynamics, which complicate the solutions in this fleld of applied physics.

We are interested in very high stagnation temperatures, and then rhemical phenomena are present; the bow shock comected with a blunt body, as necessary in re-entry problems, gives such high entropy gradients that the classical potential approach is obviously completely wong.

The relaxation times in the physical and chemical transformations of the molecular species can modify the flow properties between two extreme cases, namely the equilibrium flow and the completely frozen flow.

These are some of the difficulties encountered in hypersonic problems; other difficulties arise from consideration of the boundary layer and the local mean free path of the molerules.

We are dealing with the particular case of the continuous flow of a real gas in equilibrium, in a thin layer between shock and body. Fiscous effects are not considered; the inverse method is used. The thin shock layer theory, developed in order to calculate with a good degree 
of approximation the flow field produced by a blunt body at a very high Mach number, warrants the supposition that the shock shape is very similar to the body shape because of the small stand-off distance between the shock and the body. The intrinsic weakness of the inverse method - small changes in shock shape correspond to bigger changes in body shape - is much less important at high than at low Mach mumbers.

With a given shock shilpe, the corresponding body found does not coincide exactly with the desired body but it does correspond to a miform stream before the shock.

Many authors have considered the problem of the blunt body in supersonic and hypersonic flow during the last few years. Fan Dyke ( $\left.{ }^{7}\right)$ and Van Dyke and Gordon $\left(^{8}\right)$ make the calculations for a family of shock waves of conical shape; this gives, as Van Dyke shows, similar body shapes, at least up to the sonic point. These authors are dealing with a perfect gas.

Some modifications to Van Dyke's method are suggested by Vinokut and sanders ('1) in order to areount for different shork shapes; for enthalpy they propose the formula:

$$
h=\frac{\gamma}{\gamma-1} \frac{p}{\varrho}+A
$$

where $\gamma$ and $A$ are constants, but different before and after the shock.

Vaglio-Laurin and Ferri (1), also for a perfect gas, make the numerial calculation of the subsonic region before an arbitrary axisymmetrical body. They introduce two new indegendent rariables, one of which is related to the stream function. The stream lines, the shock line and the body are known in the plane of the new variables. It the end, the results are reduced to the physical plane.

Vaglio-Lamrin ( $\left.{ }^{2}\right)$, on the sane basis, takes into account the P.L.K. perturbation methor, consisting in stretching the coordinates in order that an alrealy known solution overlaps the field of a body having a slightly different profile.

Vaglio-Laurin and Trella $\left({ }^{3}\right)$ carried ont the calculations for many bodies, in various re-entry conditions, for a perfect gas or equilibrium gas; equilibrium equations are given in a polynomial form of the entrops in function of enthalpy and pressure. In the shock jump they express enthalpy in terms of pressure and density

$$
h=\frac{\Gamma}{\Gamma-1} \frac{\mu}{\varrho}
$$

with $\Gamma$ slightly varying along the shork. 
Our aim is to approximate the Mollier diagram in terms of a function $f(\xi ; \eta)$ of entrops and enthalpy, by which function all the thermodynamic quantities are expressed.

A pseudospheric body with a $20^{\circ}$ conic afterbody, as the "Mereury Project", is taken as an example.

II - Equations of mo'tox downstrem of JHe shock.

Let $x$ and $y$ be the curvilineas coordinates on the shock (Fig. 1).

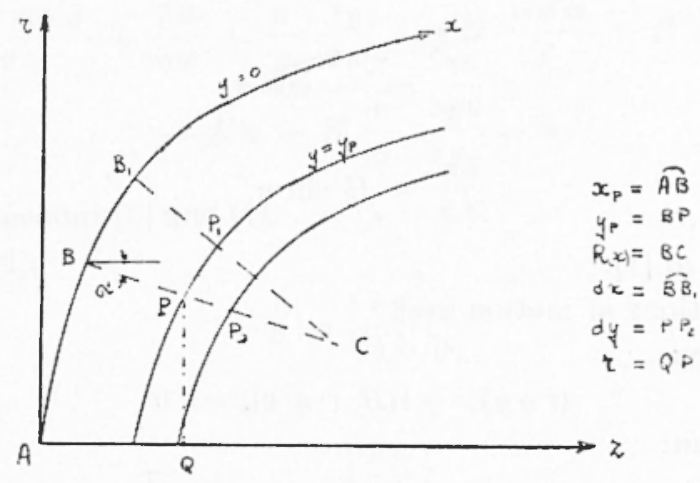

Fig. 1

Let $y=0$ be the equation of the shock wave and $I(x)$ its local curvature:

$$
K(x)=\frac{1}{R(x)}=-\left(\frac{\partial \sigma}{\partial x}\right)_{y=0}=\left(\frac{\partial \sigma^{\prime}}{\partial x}\right)_{y=0}
$$

$x$ being the curvilinear abscissa along the shock wave. The definition of the coordinates is completed stating that the line

$$
x=\text { const. }
$$

is a straight line perpendicular to the local shock wave direction, and that the $y$ coordinate of a point $P$ is the distance of $P$ from the shock wave along the line $x=$ const.

The curvature of the line $y=$ const is:

$$
\frac{1}{R(x)-y}=\frac{K(x)}{1-y K(x)}=K(x) H(x ; y)
$$

with:

$$
H(x ; y)=1-y K(x)
$$


An elementary are is given by:

or:

$$
d s=\overline{P P_{1}^{2}}+\overline{P P}_{2}^{2}
$$

$$
d s^{2}=H^{2} d x^{-}+d y^{2}
$$

We now need the distance $P Q=r$ from the symmetry axis. It is easy to see that:

$$
r=\int_{0}^{x} \sin \sigma d x-y \cos \sigma
$$

and:

$$
\begin{aligned}
& \frac{\partial r}{\partial y}=-\cos \sigma \\
& \frac{\partial r}{\partial x}=H \sin \sigma
\end{aligned}
$$

with the help of [1].

The equations of motion are:

Continuity:

$$
(r \varrho u)_{x}+(H r \varrho v)_{y}=0
$$

Momentum:

$$
\begin{aligned}
& u u_{x}+H v u_{v}-K u v=-\frac{1}{\rho} p_{x} \\
& u v_{x}+H v v_{y}+K u^{2}=-\frac{H}{\varrho} p_{y}
\end{aligned}
$$

Entropy:

$$
H \frac{\partial S^{\prime}}{\partial !}=-\frac{u}{v} \frac{\partial S}{\partial x}
$$

We choose $S$ and $h$ as thermodynamic variables; so we wish to eliminate $p$ and $\varrho$ from [8], [9] and [10].

From the known formula:

$$
T d s=d h-\frac{d p}{\varrho}
$$

we have:

$$
-\frac{1}{\rho} \frac{\partial p}{\partial x}=-\frac{\partial h}{\partial x} \div \xi \frac{\partial \aleph}{\partial x}
$$

but:

$$
h+\frac{u^{2}}{2}+\frac{v^{2}}{2}=\text { const }
$$


Thell:

$$
-\frac{1}{\varrho} \frac{\partial p}{\partial x}=u \frac{\partial u}{\partial x}+v \frac{\partial v}{\partial x}+T \frac{\partial S}{\partial x}
$$

and [9] becomes:

$$
H \frac{\partial u}{\partial y}=K u+\frac{\partial v}{\partial x}+\frac{T}{v} \frac{\partial S}{\partial x}
$$

Instead of equation [10], the energy equation [13] in closed form is preferred. We now use [8] to get $\partial v / \partial y$. Fquation [8] can be easily transformed into:

$$
\begin{gathered}
\frac{u H \sin \sigma}{r}+\frac{\partial u}{\partial x}+\frac{u}{\varrho} \frac{\partial \varrho}{\partial x}-v H \frac{\cos \sigma}{r}-H \frac{\partial v}{\partial y}- \\
-K v+H \frac{v}{\varrho} \frac{\partial \varrho}{\partial y}=0
\end{gathered}
$$

taking into account [6] and [7].

From [12]:

$$
\varrho=\left(\frac{\partial p}{\partial h}\right)_{/ s}
$$

Further:

$$
a^{2}=\left(\frac{\partial p}{\partial \varrho}\right)_{S}=\frac{\left(\frac{\partial p}{\partial h}\right)_{S}}{\left(\frac{\partial \varrho}{\partial h}\right)_{S}}==\frac{\varrho}{\left(\frac{\partial \varrho}{\partial h}\right)_{S}}
$$

or:

$$
\frac{1}{\varrho}\left(\frac{\partial}{\partial} h\right)_{s}=\frac{1}{a^{2}}
$$

Now:

$$
\frac{1}{\varrho} \frac{\partial g}{\partial x}=\frac{1}{\varrho}\left\{\frac{\partial \varrho}{\partial S} \frac{\partial S}{\partial x}-\frac{\partial Q}{\partial h} \frac{\partial h}{\partial x}\right\}
$$

and:

$$
\frac{1}{\rho} \frac{\partial \underline{Q}}{\partial x}=\frac{1}{\varrho} \frac{\partial Q}{\partial S} \frac{\partial S}{\partial x}-\frac{1}{a^{2}}\left(u-\frac{\partial u}{\partial x}+v \frac{\partial v}{\partial x}\right)
$$

using [17] and [13].

In the same way we can obtain:

$$
\frac{1}{\varrho} \frac{\partial \varrho}{\partial y}=\frac{1}{\varrho} \frac{\partial \varrho}{\partial S} \frac{\partial S}{\partial y}-\frac{1}{a^{2}}\left(u \frac{\partial u}{\partial y}+v \frac{\partial u}{\partial y}\right)
$$


If we introduce [18] and [19] into [15], the terms containing the entropy ranish by virtue of [11] and we get:

$$
\begin{gathered}
\left(1-\frac{u^{2}}{a^{2}}\right) \frac{\partial u}{\partial x}+H\left(1-\frac{v^{2}}{a^{2}}\right) \frac{\partial v}{\partial y}-\frac{u v}{a^{2}}\left(H \frac{\partial u}{\partial y}+\frac{\partial v}{\partial x}\right)- \\
-K v+H \frac{u \sin \sigma-v \cos \sigma}{r}=0
\end{gathered}
$$

We express the term $H \frac{\partial u}{\partial f}$, given by [14]:

$$
\begin{gathered}
\left(1-\frac{u^{2}}{a^{2}}\right) \frac{\partial u}{\partial x}+H\left(1-\frac{v^{2}}{a^{2}}\right) \frac{\partial v}{\partial y} \quad \frac{2 u v}{a^{2}} \frac{\partial v}{\partial x} \cdot \frac{u}{a^{2}} T \frac{\partial S}{\partial x}- \\
-\left(1+\frac{u^{2}}{a^{2}}\right) K v+H \frac{u \sin \sigma-v \cos \sigma}{r}=0
\end{gathered}
$$

On the symmetry axis:

$$
\lim _{x \rightarrow 0}\left\{H \frac{u \sin \sigma-v \cos \sigma}{r}|=| \frac{\partial u}{\partial x}-\left.K v\right|_{x=0}\right.
$$

Sote that equations [11] and [14] could be obtained directly from Croceo's theorem, written in arvilinear coordinates. Equations [14], [21], [11] and [13] contain $u ; v ; S$ and $h$ as unknowns; in an adimensional form:

$$
\begin{aligned}
& H \frac{v}{a_{o}} \frac{\partial \frac{u}{a_{o}}}{\partial y}=K \cdot \frac{u}{a_{0}} \frac{v}{a_{o}}+\frac{v}{a_{0}} \frac{\partial \frac{v}{u_{o}}}{\partial x}+\frac{\theta}{y} \frac{\partial \xi}{\partial x} \\
& \left(1-\frac{u^{2}}{a^{2}}\right) \frac{\partial \frac{u}{a_{0}}}{\partial x}+H\left(1-\frac{v^{2}}{a^{2}}\right) \frac{\partial \frac{v}{a_{0}}}{\partial y}-\dot{z} \frac{u v}{a^{2}} \frac{\partial \frac{v}{a_{0}}}{\partial x}-\frac{0}{\gamma} \frac{u}{a_{0}} \frac{a_{0}^{2}}{a^{2}} \frac{\partial \xi}{\partial x}- \\
& -\left(1+\frac{u^{2}}{a^{2}}\right) K \frac{v}{a_{o}}+H\left(\frac{u}{a_{0}} \sin \sigma-\frac{v}{a_{01}} \cos \sigma / /\right) \cdot r=0 \\
& \frac{2}{y} \eta+\frac{u^{2}}{a_{0}^{2}}+\frac{v^{2}}{u_{0}^{2}}=\frac{2}{\gamma} \eta_{\max } \\
& H \frac{\varepsilon}{a_{0}} \frac{\partial \xi}{\partial y}=-\frac{u}{a_{0}} \frac{\partial \xi}{\partial x}
\end{aligned}
$$




\section{III - DETERMIN.TION OF THE FUXCTIO $f(\xi ; \eta)$}

For a perfect gas we have, from [12]:

$$
\begin{gathered}
d l=\varrho d h-p \frac{d s}{R_{o}} \\
\left(\frac{\partial p}{\partial s}\right)_{h}=-\frac{p}{R_{o}}
\end{gathered}
$$

and, after integration:

$$
\frac{l}{y_{0}}=e^{-\frac{s}{\pi_{0}}} f(h)
$$

But:

$$
\left(\frac{\partial \mu}{\partial h}\right)_{S}=\varrho=p_{0} e^{-\frac{s}{R_{0}}} f^{\prime}(h)
$$

and, dividing:

$$
\frac{f(h)}{f^{\prime}(h)}=\frac{p}{\varrho}=R_{n} \cdot r=\frac{\gamma-1}{\gamma} h
$$

Then:

$$
f(h)=h^{3.5}
$$

for $\gamma=1.4$

The result is that, for a perfert gas:

$$
\frac{l^{\prime}}{p_{0}}=\left(\frac{h}{h_{0}}\right)^{3.5} e^{-\frac{s-s_{0}}{k_{0}}}
$$

If we introduce adimensional quantities:

$$
\frac{p}{p_{0}}=F=\left(b_{0}+b_{1} \eta\right)^{3,5} e^{-\breve{\xi}}
$$

where $\eta$ and $\xi$ are the arlimensional enthalpy and entropy differences evaluated from a given state.

For the real case of air in thermodynamic equilibrium we put, in analogy with [26]:

$$
F=C_{0} 10^{-C_{1} \frac{\zeta}{4}-c_{2} \zeta^{2}}[f(\xi ; \eta)]^{\prime \prime}
$$

whele $C_{0} ; C_{1} ; C_{2}$ and $n$ are constants.

For function $f(\xi ; \eta)$ we assume, in analogy with [28]:

$$
f(\xi ; \eta)=g_{0}(\xi)+\eta g_{1}(\xi)+\eta^{2} g_{2}(\xi)
$$


Functions $g_{k}(\xi)$ are given in order to approximate the Mollier diagram for equilibrium air [22] in the field:

$$
\begin{gathered}
280<\frac{h}{R_{o} T_{o}}<360 \\
48<\frac{S}{R_{o}}<52
\end{gathered}
$$

In this field $f(\xi ; \eta)$ has been evaluated from [29], [33] and

$$
\frac{F}{G}=\frac{p / p_{o}}{g / o_{o}}=\ddot{\ell} ?-g \frac{T}{T_{o}}=\frac{f(\xi ; \eta)}{n f_{r .}(\xi ; \eta)}
$$

Dropping very few terms, this gives:

$$
f(\xi ; \eta)=\left(1+b_{1} \xi\right)+\left(b_{2}+b_{1} \xi\right) \cdot \eta+b_{i} \xi \eta^{2}
$$

and:

$$
\begin{aligned}
& n=8.2192 \\
& b_{1}=-0.00416 \\
& b_{2}=0.0032706 \\
& b_{4}=0.0000723 \\
& b_{7}=0.98 .10^{-7}
\end{aligned}
$$

The valne of $n$ is determined so as to give $f(o ; \eta)$ a linear expression in $\eta$

$$
f(o ; \eta)=1+0.0032706 \eta
$$

as is the case in a perfect gas, for any $\xi$, from [28].

Writing [16] in arlimensional form, we now obtain from [29]:

$$
G=\frac{\varrho}{\varrho_{o}}=\frac{\partial F}{\partial \eta}=C_{o} n \cdot 10^{-C_{1} \dot{\xi}-C_{2} \dot{\sigma}^{2}}[f(\xi ; \eta)]^{n-1} \frac{\partial f}{\partial \eta}
$$

The speed of sound is given by [17], or

$$
\frac{R_{n} T_{o}}{a^{2}}=\frac{a_{0}^{2}}{\gamma a^{2}}=\frac{1}{G}\left(\frac{\partial G}{\partial \eta}\right)_{\xi}=\left(\frac{\partial \lg G}{\partial \eta}\right)
$$

and:

$$
\frac{a_{n}^{2}}{a^{-}}=(n-1) \gamma \frac{\stackrel{f}{\prime \prime}(\xi ; \eta)}{f(\xi ; \eta)}+\gamma^{\prime} \frac{f_{m}(\xi ; \eta)}{f_{\eta}(\xi ; \eta)}
$$

We must now express the temperature. 
From [12], we get:

$$
T=\left(\frac{\partial h}{\partial S}\right)_{s}=-\frac{\left(\frac{\partial p}{\partial S}\right)_{h h}}{\left(\frac{\partial p}{\partial h}\right)_{S}}
$$

or:

$$
0=\frac{-\frac{\partial F}{\partial \xi}}{\frac{\partial F}{\partial \eta}}=-\frac{\frac{\partial F}{\partial \xi}}{G}
$$

and from [33] and [29]:

$$
\theta=\frac{1}{f_{\eta}(\xi, \eta j)}\left\{\frac{2095 \Omega 5}{n}\left(C_{1}+2 C_{2}^{\prime} \xi\right) f(\xi ; \eta)-f_{\xi}(\xi ; \eta)\right.
$$

Note that from [35], we can get:

$$
\frac{1}{Z}=\frac{\varrho R_{o} T}{p}-\frac{G \theta}{F}--\frac{\partial \lg F}{\partial \xi}
$$

or, from [29]:

$$
\frac{1}{Z}=2.302585\left(C_{1}+2 C_{2} \xi\right)-\frac{n f_{\xi}(\xi ; \eta)}{f(\xi ; \eta)}
$$

The validity of [38] for $\eta=0$ and:

$$
48<\xi<52
$$

gives:

$$
\left\{\begin{array}{l}
C_{1}=0.2675 \\
C_{0}=-0.000922
\end{array}\right.
$$

The constant $C_{0}$ is given by [29]:

$$
C_{0}=F(O ; O)=0.5217
$$

Equation [29] is then completely specified. Equations [33], [36] and [3t] give the density, the temperature and the velocity of sound. Equations [36] and [34] will be used in the field; equations [29] and [33] will be used on the shock, to get initial conditions. Formulae [29], [33], [36] and [3t] have been controlled for values of $\xi$ and $\eta$ other than those used for calculating coefficients: the agreement is within $2-3$ percent. It could be increased by taking into account a third order term $\eta^{3}$, in [30]. 
IV - INITAL CONDITIONS ON THE SHOCK.

On the shock (r. Fig. 2 below), we have:

$$
\begin{aligned}
& \frac{v}{r_{\infty}}=\varepsilon=\frac{\varrho_{\infty}}{\varrho} \\
& p \quad=p_{\infty}+\varrho_{\infty} v_{\infty}^{2}(1-\varepsilon) \\
& h \quad=h_{\infty}+\frac{1}{2} v_{\infty}^{2}\left(1-\varepsilon^{2}\right) \\
& u=u_{\infty}
\end{aligned}
$$

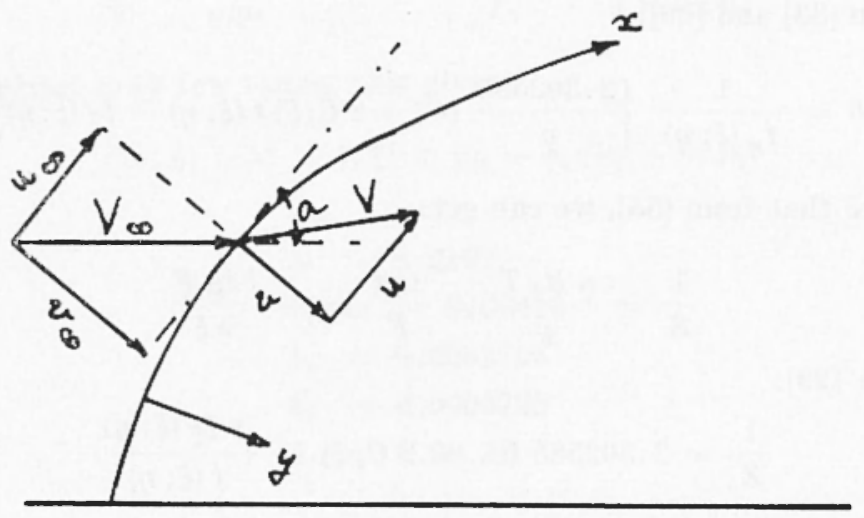

Fig. 2

Putting $v_{\infty}=r_{\infty}$ sin $\sigma$ and noting $\left[{ }^{22}\right]$ that at an altitude of $60 \mathrm{~km}$ $T_{\infty}=T_{\circ}$ or $\theta_{\infty}=1$ :

$$
\begin{aligned}
(i & =\frac{G_{\infty}}{\varepsilon} \\
F & =F_{\infty}+1.4 G_{\infty} M_{\infty}^{2}(1-\varepsilon) \sin ^{2} \sigma \\
\eta & =0.7 M_{\infty}^{2}\left(1-\varepsilon^{-}\right) \sin ^{2} \sigma-296.5 \\
\frac{f(\xi ; \eta)}{n f_{\eta}(\xi ; \eta)} & =\frac{F}{G} \\
G & =G(\xi ; \eta) \\
\varepsilon^{\prime} & =\frac{(\dot{G})}{G(\xi ; \eta)}
\end{aligned}
$$

with:

$$
\left\{\begin{array}{l}
G_{\infty}=F_{\infty}=3.5 \cdot 10^{-4} \\
H_{\infty}=22.6 \\
F_{\infty}=7,500 \mathrm{~m} / \mathrm{sec} .
\end{array} \quad 900 \geqslant \sigma \geqslant 600\right.
$$


Equations [45] to [50] alle iterited nutil we get $\varepsilon=\varepsilon^{\prime}$ (v. Fig. 3 below).

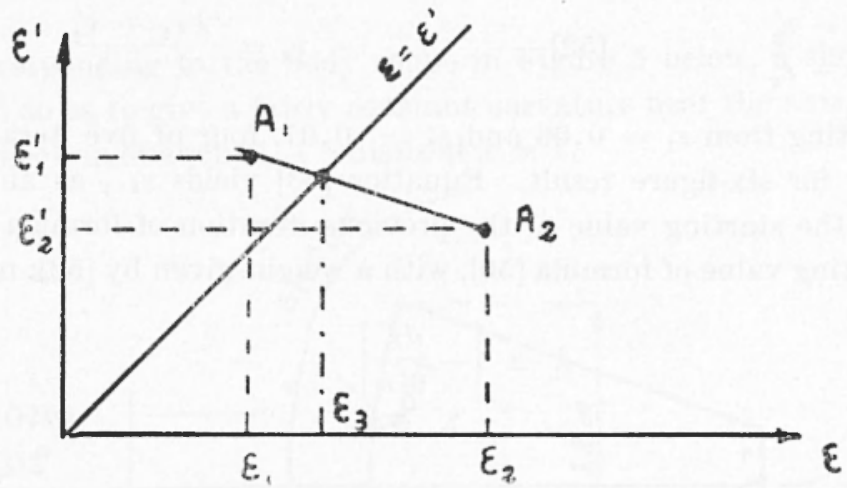

Fig. 3

Tahle no. 1

\begin{tabular}{|c|c|c|c|c|c|}
\hline$\sigma^{\prime}$ & $\varepsilon$ & y & $\xi$ & $u / a_{0}$ & $v / a_{0}$ \\
\hline 00 & 0.0604011 & 59.727650 & 3.7321361 & 0 & $1.36+483$ \\
\hline 10 & 0.0604070 & 59.618930 & 3.7282209 & 0.394202 & 1. $36+277$ \\
\hline $2^{\circ}$ & 0.0604246 & 59.292750 & 3.7164604 & 0.788403 & $1.36+181$ \\
\hline $3^{0}$ & $0.060+539$ & 58.749670 & $3.69687+9$ & 1.182379 & 1. 363804 \\
\hline $4^{\circ}$ & 0.0604951 & 57.990230 & 3.6094715 & 1.575900 & 1.363272 \\
\hline $5^{\circ}$ & 0.0605481 & $57.015+10$ & $3.633+2657$ & 1.968885 & 1.3625999 \\
\hline $6^{0}$ & 0.0606131 & 55.826350 & 3.5912786 & 2.361370 & 1.361766 \\
\hline $7^{\circ}$ & 0.06069001 & 54.424600 & 3.5405360 & 2.753090 & 1.360795 \\
\hline 80 & 0.0607792 & $52.8117+0$ & 3.48206692 & 3.143900 & 1. 3596660 \\
\hline $9^{\circ}$ & 0.0608805 & 50.989850 & 3.4159125 & 3.533810 & 1.358371 \\
\hline $10^{\circ}$ & 0.06099942 & 48.961110 & 3.3421087 & 3.922820 & $1.356944 \mathrm{j}$ \\
\hline 110 & 0.0611205 & $4(0.727950$ & 3.2067018 & +.310470 & 1.355359 \\
\hline 120 & 0.0612595 & 44.293180 & $3.1717+62$ & +.696760 & $1.353+27$ \\
\hline $13^{\circ}$ & $0.061+112$ & 41.659690 & 3.0752968 & 5.081701 & $1.3517+7$ \\
\hline $14^{\circ}$ & 0.0615762 & $38.8307+0$ & $2.971+190$ & $5 .+65060$ & 1.349715 \\
\hline $15^{\circ}$ & 0.06117544 & 35.809770 & 2.8601824 & 5.846837 & 1.347525 \\
\hline $16^{\circ}$ & 0.06119463 & 32.600450 & 2.74166355 & (j. 2268007 & 1.34517 \\
\hline $17^{\circ}$ & 0.0621523 & 29.2066690 & $2.6159+85$ & $6.6047+3$ & 1. 342686 \\
\hline $18^{\circ}$ & 0.0623723 & 25.632040 & $2 .+831201$ & 6.980873 & 1.3400506 \\
\hline 190 & 0.0626071 & $21.88269_{0}$ & 2.34328165 & $7.3547+3$ & 1.337265 \\
\hline 200 & 0.0628568 & 17.961380 & $2.1965+30$ & 7.726355 & 1. 334320 \\
\hline $21^{\circ}$ & 0.0631219 & 13.873460 & 2.0433010 & 8.095707 & 1.331235 \\
\hline 220 & 0.0634030 & 9.623940 & 1.8525080 & 8.402575 & 1.327997 \\
\hline $23^{\circ}$ & 0.0637003 & 5. 218020 & 1.7160652 & 5.826731 & $1.324(311$ \\
\hline $24^{\circ}$ & $0.064+0146$ & 0.601080 & 1.5429261 & $9.188+03$ & 1.321096 \\
\hline $25^{\circ}$ & $0.064+3466$ & 4.041360 & 1. 3635380 & 9.547138 & $1.317+24$ \\
\hline $26^{\circ}$ & $0.06+69668$ & - 8.883560 & 1.1780605 & 9.902936 & 1.313607 \\
\hline $27^{\circ}$ & 0.06506600 & -13.859630 & 0.986666399 & 10.255798 & 1. 3096604 \\
\hline 280 & $0.0155+549$ & -18.963480 & 0.7895265 & $10.605+90$ & 1.305570 \\
\hline $299^{\circ}$ & 0.0658645 & -24.188910 & $0.5868+09$ & 10.962033 & 1.301349 \\
\hline 300 & 0.0662957 & $\ldots-29.5295+0$ & 0.3788113 & 11.295180 & 1.297000 \\
\hline
\end{tabular}


Two simple formulate are used instead of the graphical method illustrated in Fig. 3:
$\lambda=\frac{\varepsilon_{1}^{\prime}-\varepsilon_{2}^{\prime}}{\varepsilon_{2}-\varepsilon_{1}}$$$
\varepsilon_{3}=\frac{\lambda \varepsilon_{2}+\varepsilon_{2}^{\prime}}{\lambda+1}
$$

Starting from $\varepsilon_{1}=0.06$ and $\varepsilon_{2}=0.07$, four or five iterations are sufficient for six-figure result. Equation [53] yields $\varepsilon_{i+1}$ as an average between the starting value of the previous iteration of formula [45] and the resulting value of formula [50], with a weight given by [52]: note that:

$$
\lambda \simeq\left[-\frac{d \varepsilon^{\prime}}{d \varepsilon}\right]_{\varepsilon^{\prime}=\varepsilon}
$$

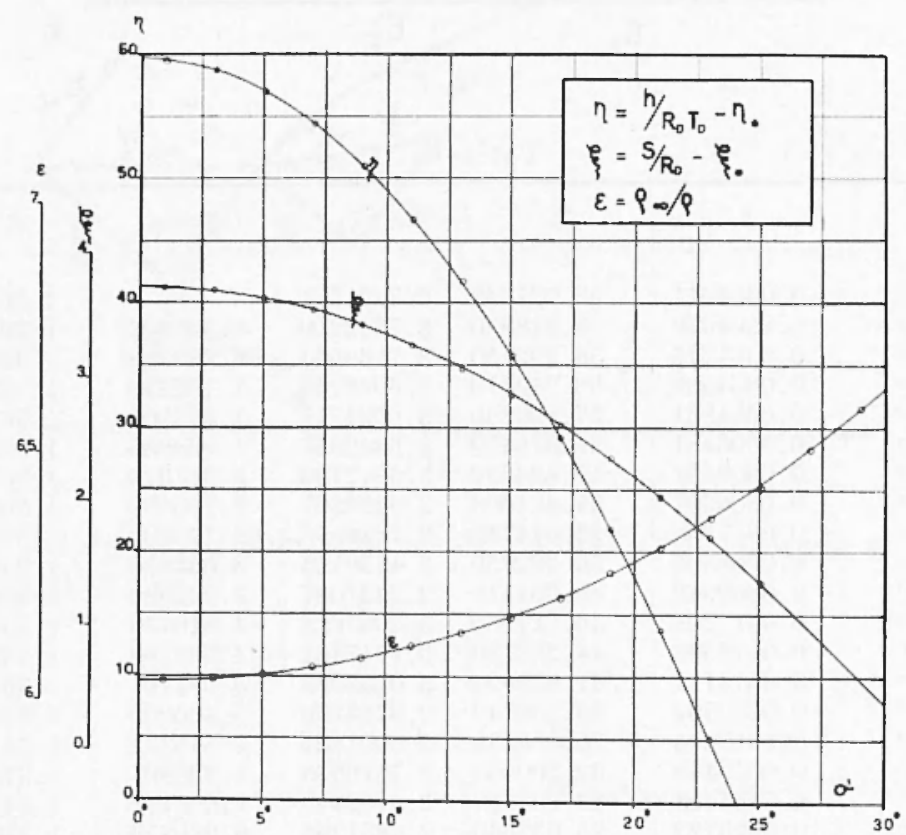

Fig. 4

When the true value of $\varepsilon$ is obtained, we get:

$$
\left\{\begin{array}{l}
v=\varepsilon v_{s=}=\varepsilon \bar{v}_{z:} \sin \sigma \\
u=\omega_{\infty}=V_{\infty} \cos \sigma
\end{array}\right.
$$

Table I contains the results in function of $\sigma^{\prime}=\frac{\pi}{2}-\sigma$. Fig. 4 shows $\varepsilon, \xi, \eta$ along the shock. 
V - THE SHAPE OF THE SHOCK.

Corresponding to the body shape in Figure 5 below, a shock shape is chosen so as to give a fairly constant curvature near the axis, followed by an inflection of about 1.4 radians at $x \simeq 1$.

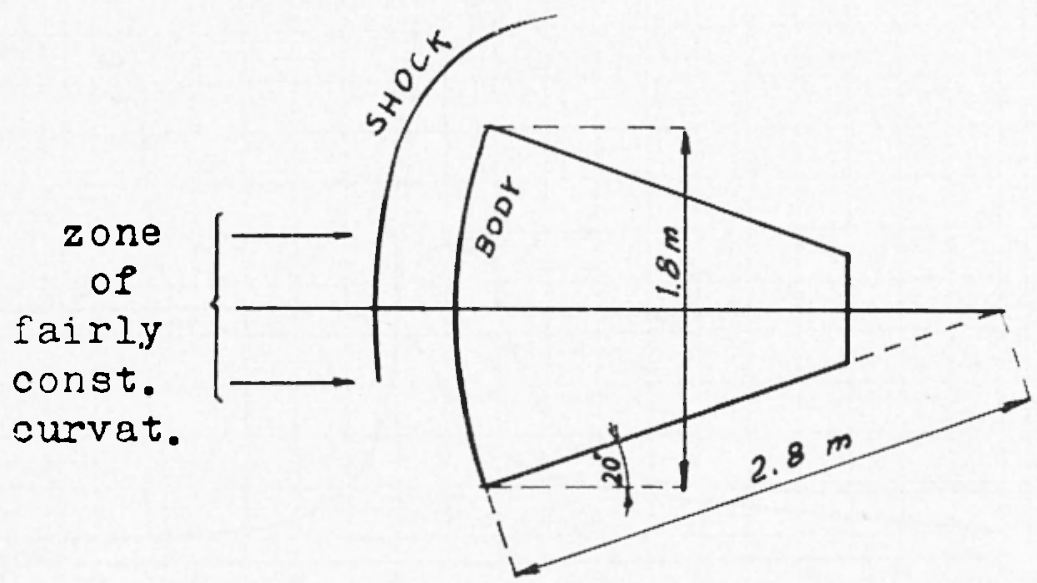

Fig. 5

The type of function representing the curvature is then:

$$
K(x)=\frac{1}{3-3 b x^{4}+b x^{8}}
$$

which has a maximum just at $x=\sqrt[4]{1.5} \simeq 1.1$. The condition that the total inflection be about 1.4 radians implies $b=1.25$ (Fig. 6). From [50] we then get:

$$
\int_{0}^{x} K(x) d x=\sigma^{\prime}=\frac{\pi}{2}-\sigma
$$

By reversal of this formula $x$ is determined as a function of $\sigma$ or $\sigma^{\prime}$. Table II and Fig. 7 show $x$ and $K$.

The shape of the shock is then given by (v. Fig. 1):

$$
\begin{aligned}
& r=\int_{0}^{x} \sin \sigma d x=\int_{0}^{\sigma^{\prime}} \cos \sigma^{\prime} \frac{d \sigma^{\prime}}{\hbar\left(\sigma^{\prime}\right)} \\
& z=\int_{0}^{x} \cos \sigma d x=\int_{0}^{\sigma^{\prime}} \sin \sigma^{\prime} \frac{d \sigma^{\prime}}{h\left(\sigma^{\prime}\right)}
\end{aligned}
$$




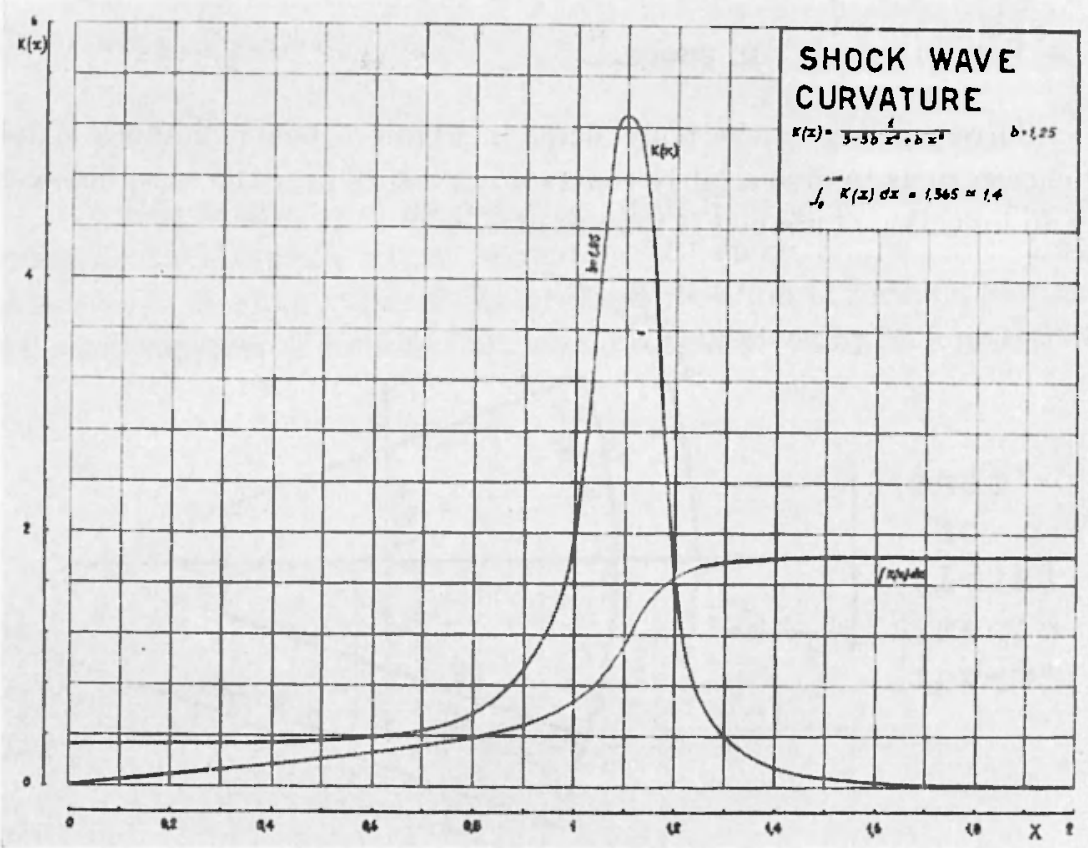

Fig. 6

Table no. 2

\begin{tabular}{|c|c|c|c|c|c|}
\hline$\sigma^{\prime}$ & $I$ & K $\left(\sigma^{\prime}\right)$ & $\sigma^{\prime}$ & $T$ & $\hbar\left(\sigma^{\prime}\right)$ \\
\hline 0 & 0.000 & 0.333333 & $16 \mathrm{i}$ & 0.760713 & 0.530544 \\
\hline 1 & 0.05236 & 0.333336 & 17 & 0.792204 & 0.582585 \\
\hline 2 & 0.104716 & 0.333383 & 18 & $0.82067 \mathrm{~s}$ & 0.642483 \\
\hline 3 & 0.156967 & 0.333383 & 19 & 0.846574 & 0.712626 \\
\hline 4 & 0.209410 & 0.333413 & 20 & $0.8697+1$ & 0.791277 \\
\hline 5 & 0.261565 & (). 335295 & 21 & $0.8907+6$ & 0.881625 \\
\hline 6 & 0.313515 & $0.337+11$ & 22 & 0.909424 & 0.980279 \\
\hline 7 & 0.364956 & 0.340852 & 23 & $0.926+11$ & 1.002132 \\
\hline 8 & 0.415808 & 0. 346165 & 24 & $0.9+1+71$ & 1.211032 \\
\hline 9 & 0.465671 & $0.35+281$ & 25 & 0.95528 & 1.344798 \\
\hline 10 & $0.51+263$ & $0.36+453$ & 26 & 0.9067502 & 1. 483055 \\
\hline II & 0.561300 & 0.378859 & 27 & 0.978549 & 1.638133 \\
\hline 12 & 0.606286 & 0.397503 & 28 & 0.988874 & 1. 793741 \\
\hline 13 & 0.649008 & 0.421324 & 29 & 0.098330 & 1.968372 \\
\hline 14 & 0.689069 & 0.450404 & 30 & 1.0066601 & 2. 138292 \\
\hline 15 & $0.726+14$ & 0.487209 & & & \\
\hline
\end{tabular}




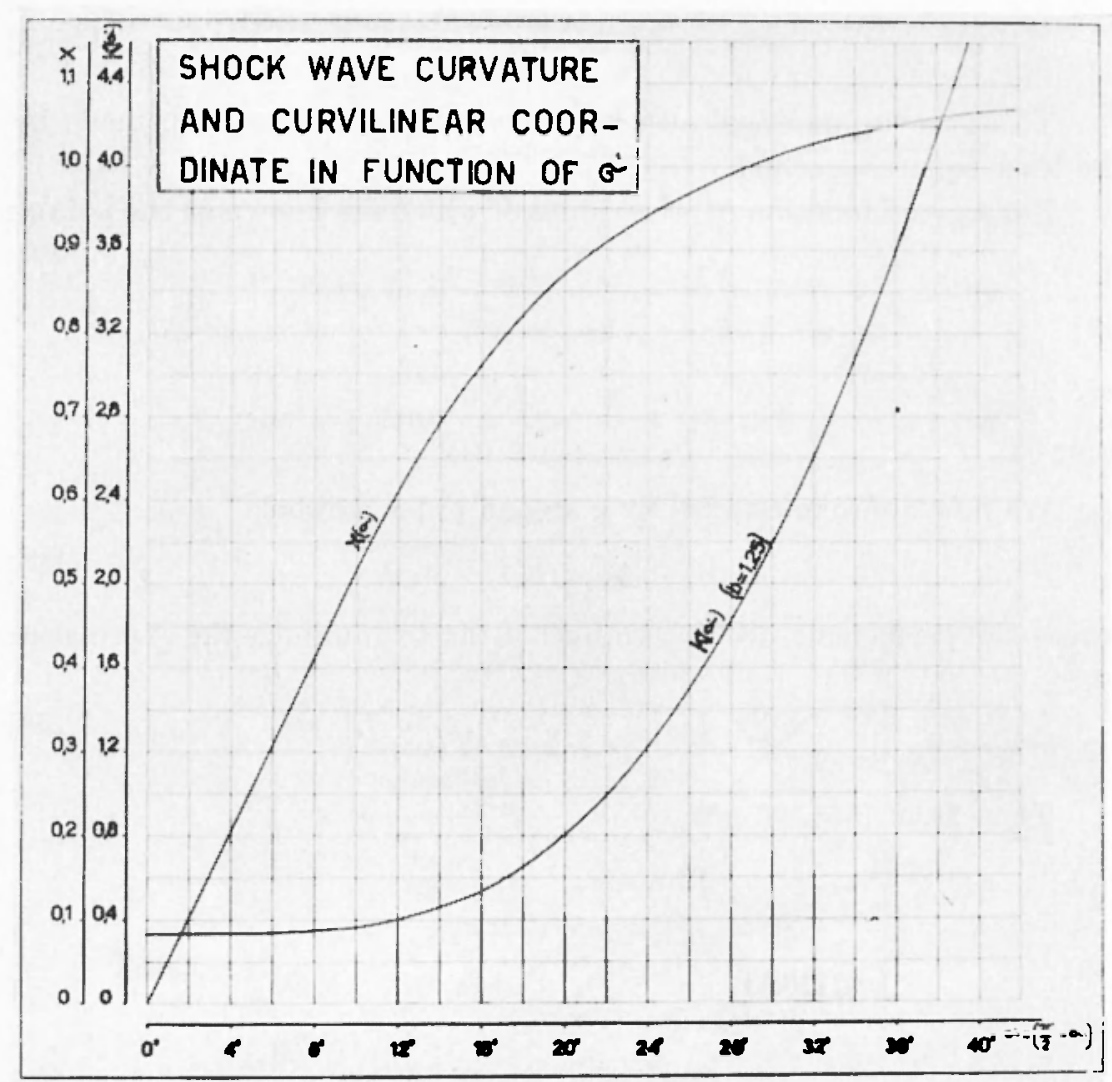

Fig. -

VI - EVALUATIOx OF THE FIELI BEILND THE SHOCK.

The evaluation of the aerothermodymamic field is done by a stepby-step procedure.

Consider equations [\%2] to [25].

If we suppose all variables along the line to be known, it is possible to calculate the three $x$-derivatives as:

$$
\begin{aligned}
& \left(\frac{\partial \xi}{\partial x}\right)_{y} \equiv K\left(-\frac{\partial \xi}{\partial \sigma^{\prime}}\right)_{y} \\
& \left(\frac{\partial u / a_{o}}{\partial x}\right)_{y} \equiv K\left(\frac{\partial u / a_{o}}{\partial \sigma^{\prime}}\right)
\end{aligned}
$$




$$
\left(\frac{\partial v / a_{o}}{\partial x}\right)_{y}=K \frac{\partial v / a_{o}}{\partial \sigma^{\prime}}
$$

To improve numerical acculacy this calculation has been made by the least squares method.

For a given function $f(t)$ the values of which are known at the points:

let:

$$
\begin{gathered}
t_{-2}=-2 h ; \quad t_{-1}=-h ; \quad t_{0}=0 \\
t_{1}=\quad h ; \quad t_{2}=2 h \\
f_{i}=f\left(t_{i}\right) \\
f_{i}^{\prime}=f^{\prime}\left(t_{i}\right)
\end{gathered}
$$

and:

We now approximate $f(t)$ by a second order parabolat

$$
f(t)=a_{o}+a_{1} t+a_{2} t-
$$

where the coefficients are determined so as to minimize the expression:

$$
\sum_{i=-2}^{2}\left[a_{o}+a_{1} t_{i}+a_{2} t_{i}-f_{i}\right]^{2}
$$

Then from $[56]$ we gret:

$$
\begin{aligned}
& f_{o}^{\prime}=a_{1} \\
& f_{1}^{\prime}=a_{1}+2 a_{2} h \\
& f_{2}^{\prime}=a_{1}+4 a_{2} h
\end{aligned}
$$

or:

$$
\begin{gathered}
f_{o}^{\prime}=\frac{2 f_{-}+f_{1}-f_{1}-2 f_{-2}}{10 h} \\
f_{1}^{\prime}=\frac{1}{70 h_{h}}\left\{34 f_{2}-3 f_{1}-20 f_{0}-1 \tau f_{-1}+6 f_{-2}\right\} \\
f_{2}^{\prime}=\frac{1}{70 ?}\left\{54 f_{2}-13 f_{1}-40 f_{0}-27 f_{-1}+26 f_{-2}\right\}
\end{gathered}
$$

We took 31 points on the shock at $\sigma^{\prime}=0^{\circ} ; 1^{\circ} ; 2^{\circ} \ldots .30^{\circ}$. The $x$ derivatives are evaluated, as in [56], by means of $\sigma^{\prime}$-derivatives, which are given by [59], for ordinary points, and by [60] [61] for the last two points. This has the effect of slightly smoothing the process as the finite difference scheme is based on minimizing the error of the locally approximating parabola. The step in $\mathrm{y}$ was $\Delta y=0.005$ for $0<y<$ 0.08 and $\Delta y=0.002$ for $0.08<y<0.12$ near the body.

I second smoothing procedure was necessary along $y=0.04$ and later on $y=0.08$ and $y=0.10$. 
This smoothing procedure was carried out by means of [5i], that is:

$$
\begin{aligned}
& {\left[f_{0}\right]_{\text {smooth }}=a_{0}} \\
& {\left[f_{1}\right]_{\text {smooll }}=a_{n}+a_{1} h+a_{2} h^{2}} \\
& {\left[f_{2}\right]_{\text {smootl }} \simeq a_{n}+2 a_{1} h+ \pm a_{2} h^{2}}
\end{aligned}
$$

(1):

$$
\begin{aligned}
& {\left[f_{0}\right]_{\text {smooth }}=\frac{1}{35}\left\{-3 f_{2}+12 f_{1}+17 f_{0}+12 f_{-2}\right\}} \\
& {\left[f_{1}\right]_{\text {smootl }}=\frac{1}{35}\left\{9 f_{2}+13 f_{1}+12 f_{0}+6 f_{-2}-5 f_{-3}{ }^{-3} f_{-2}\right\}} \\
& {\left[f_{2}\right]_{\text {smootl }}=\frac{1}{35}\left\{31 f_{2}+9 f_{1}-3 f_{0}-5 f_{-1}+3 f_{-9}\right\}}
\end{aligned}
$$

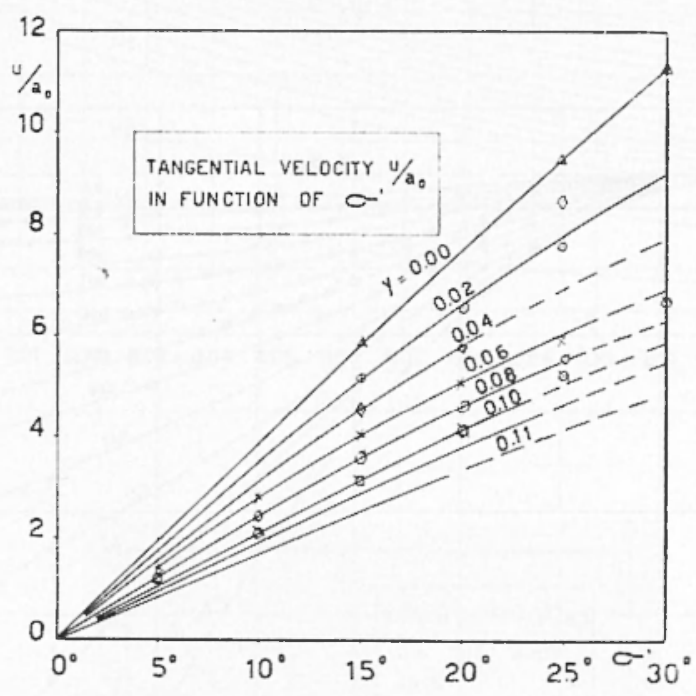

Figr. 8

Fig. 8, Fig. 9 and Fig. 10 show the two velocity components and the entropy in the field. Fig. 11 shows the Mach number: the sonice line is given by an intersection $M=1$. Fig. 11 is made np with the help of [34]. Fig. 12 and 13 give the shape of the body: $v=0$ on the axis and $\xi=\xi_{\text {max }}$ along $\sigma^{\prime}=$ ronst $>0$. In the same Fig. 13 we find the stream lines as constant-g-lines. Fig. 14 gires the body shape. In curvilinear coordinates the value of $y$ varies, but very little, from .125 on the axis to .128 at the sonic point.

Figures 15 and 16 give pressure and temperature on the body. 


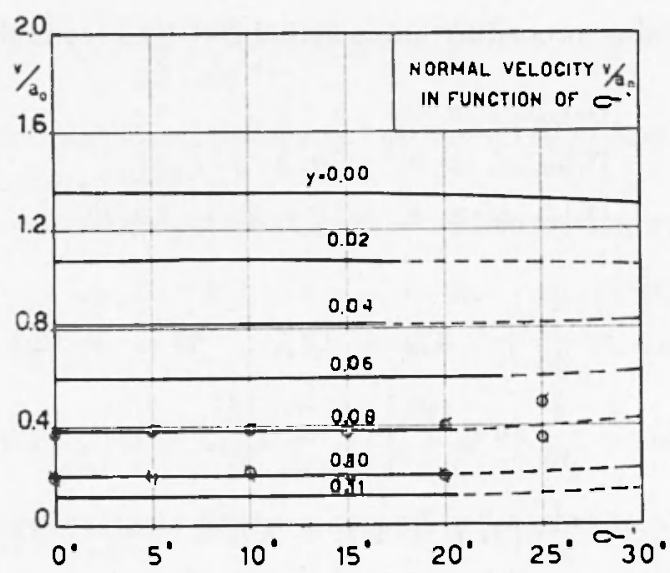

Fig. 9

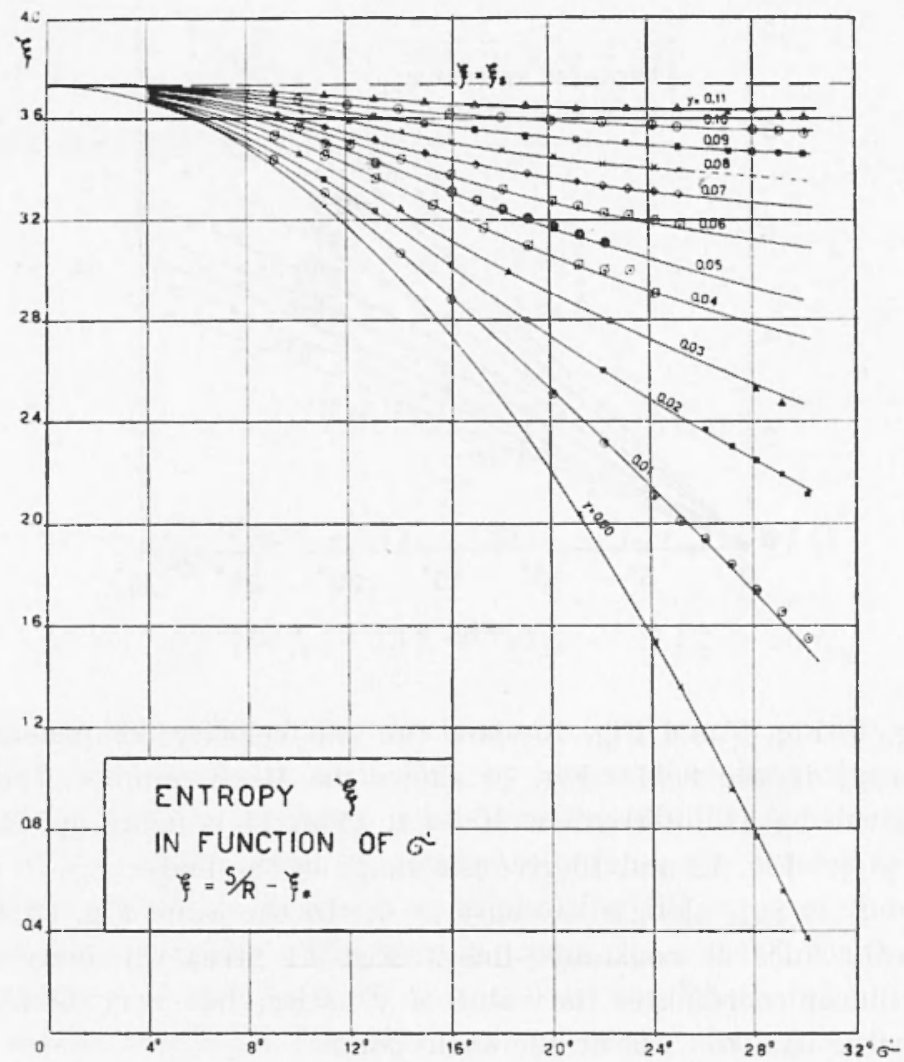

Fig. 10 
Figure 17 and 18 represent them in function of $r$. The integration of the pressure gives a diag coefficient of:

$$
C_{D}=1.71
$$

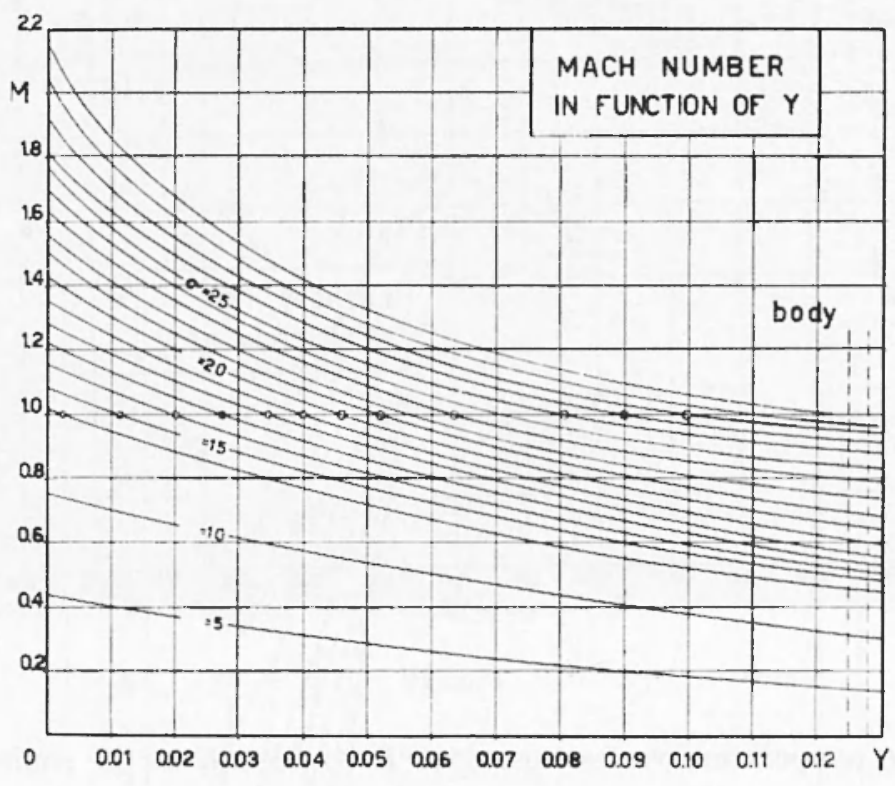

Fig. 11

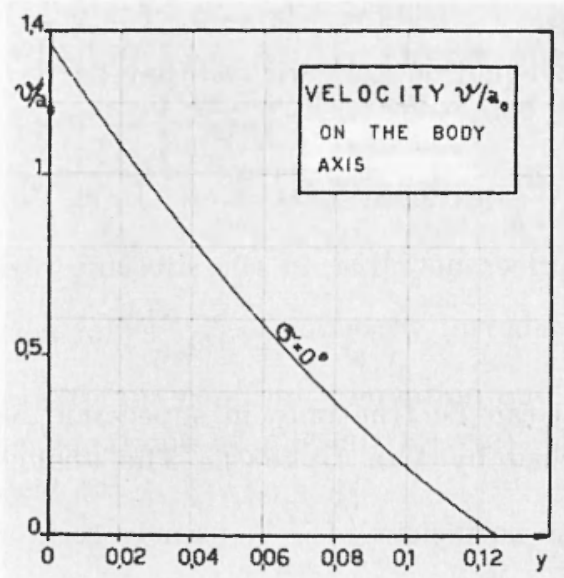

Figr. 12 


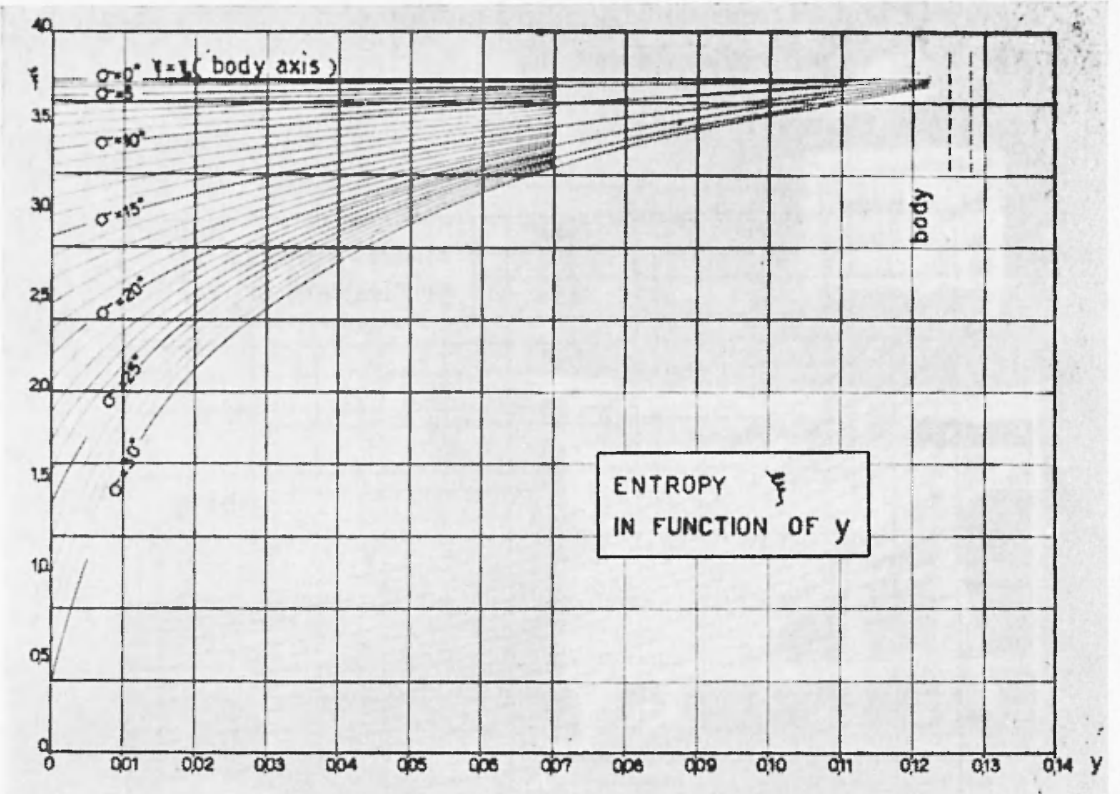

Fig. 13

The temperature varies from $6520 \% K$ to $6390 \% \mathrm{~K}$ at the sonic point.

VII - DIVISIOX BY ZERO IN COMPUTING $\frac{\partial v}{\partial y}$ NND $\frac{\partial \xi}{\partial y}$.

If we consider equation [23], we see that if:

$$
v= \pm a
$$

the coefficient of $\frac{\partial v}{\partial y}$ is zero.

This question does not arise in the subsonic region where, by definition:

$$
\sqrt{u^{2}+v^{2}} \leqslant a
$$

Condition [66] ean be true only in supersonic regions. The same conclusion is reached by Van Dyke $\left(^{8}\right)$. The analogous term in Van Dyke's work is the coefficient of $\frac{Q_{n}}{\varrho}$, namely tem $D$.

Figure 19 below, reproduced from Van Dyke's study, shows line $D=0$. 
Van Dyke in his study seeks an explanation as to whether line $D=0$ has any simple physical significance.

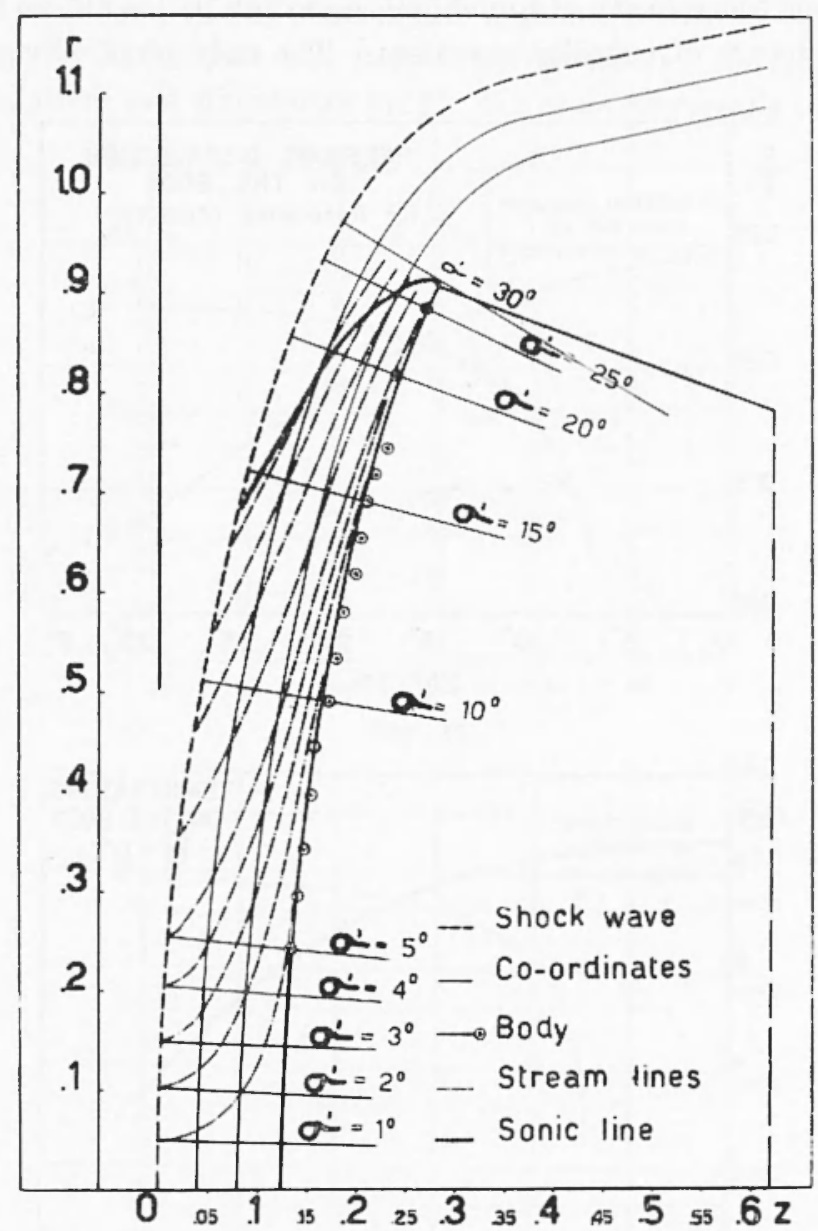

Fig. 14

As the equation for $\frac{Q_{i}}{\varrho}$ is obtained by introducing a stream function in order to satisfy the continuity equation and by eliminating the pressure from the equations of motion, we may infer that condition $D=0$ is equivalent to:

$$
\left(1-\frac{v^{2}}{c^{2}}\right)=0
$$

which is the coefficient of $\frac{\partial v}{\partial !}$ in the continuity equation [237. If this is 
so, we may say that the $D=0$ line has, but not completely, a physical significance: it would be a line along which one of the velocity components equals the velocity of sound [67]. Equation [67] is not an invariable one in a change of roordinate system. The onle physically significant

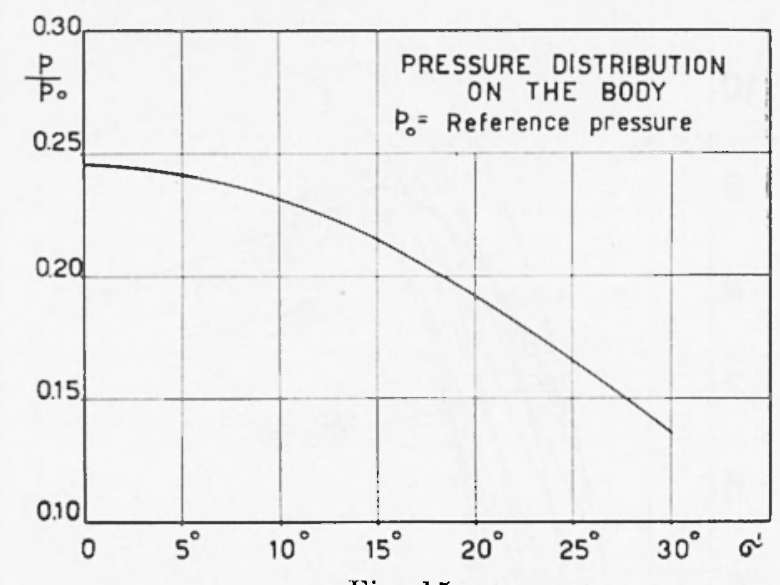

Fig. 15

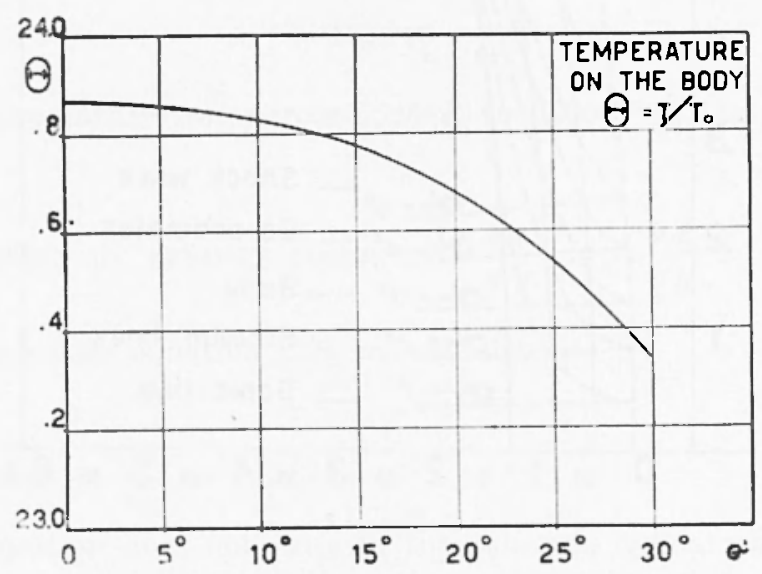

Fir. 16

thing we can infer is that the line in question lies entirely within the supersonic regrion.

We can conclude that this difficulty arises only when we are well in the supersonic region, where the method of the characteristics must be applied. Moreover, in the case of a very blunt shock shape chosen in order to get a sharp-ended blunt body, as in Fig. 5, the body profile 
rums about parallel to the shork profile and romponent $v$ is about zero along the body.

This condition, on the contrary, is negative if solving equation [25] for entropy. Component $v$ is indeed the coefficient of $\partial \xi / \partial u$ : when it vanisches, there is a singularity for $\xi$. We must necessarily infer that at

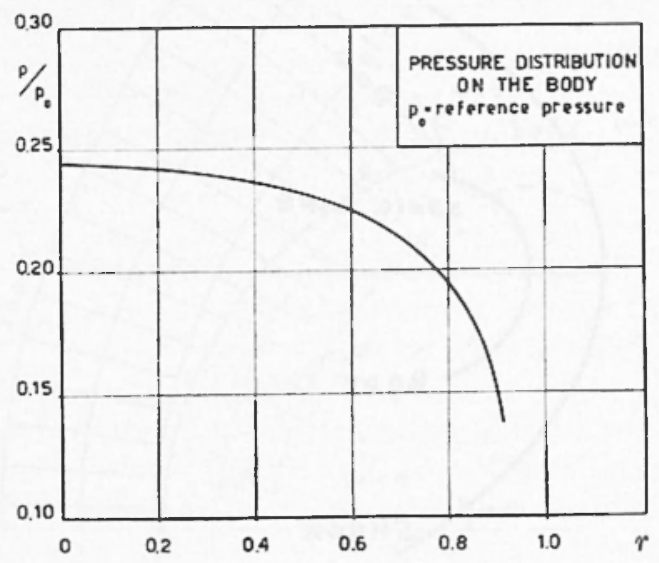

Fig. 17

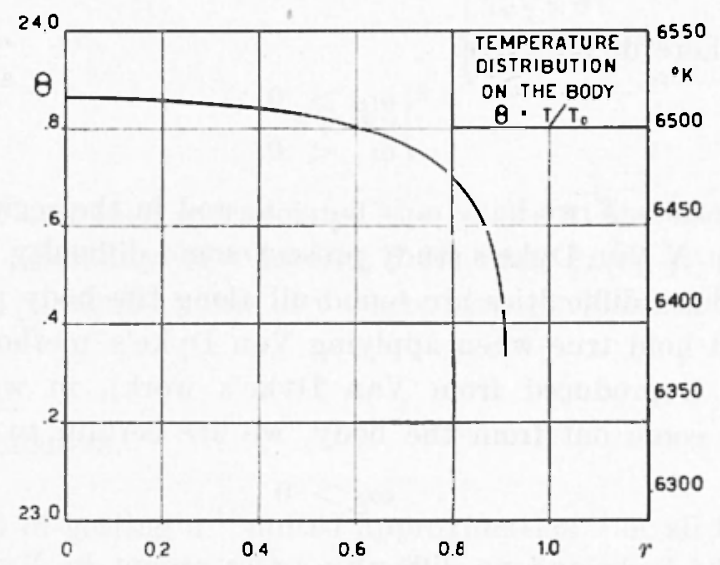

Fig. 18

the same station the term $\partial \xi / \partial x$ vanishes too and the singularity is purely mathematical. If we consider the quoted work of Van Iylie, we sece something analogous, the "bad" coefficient being:

$$
\left(\omega+\frac{\xi \omega \xi}{2}\right)
$$


where $\omega$ is related to the stream function $\psi:(1)=\frac{2 w}{\xi^{2}}$ and $\xi$ is a curvilinear roordinate.

Van Dyke states that the quantity [68] camnot vanisle, except in points such as $A$ (Fig. 20 below) internal to the body profile.

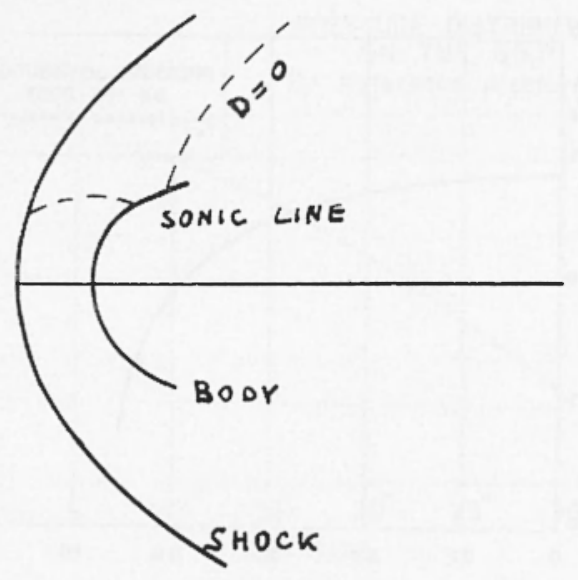

Fig. 19

Only there do we have:

$$
\left\{\begin{array}{l}
\omega \xi \\
\vdots \omega
\end{array}\right.
$$

On the nose $N$ we have $\omega=\omega_{\xi}=0$, and in the region of the stagnation point $N$ Van Dyke's study present some difficulty. In our case, however, these difficulties are found all along the body profile and the same would hold true when applying Van Dyke's method: in the ease of Fig. 20 (reproduced from Van Dyke's work), in which the lines $\eta=$ const come out from the body, we are certain to have:

$$
\text { (1) } \xi>0
$$

all along the body and no difficulty arises except in $N$. In a case like that of the present study, in which the body profile is very near to a coordinate line, we should get:

$$
\text { (1) } \simeq 0
$$

along the body, and necessarily, if the $(1)=0$ line is also an $\eta$ - const line:

$$
\text { (1) } \leqslant \simeq 0
$$


so that [68] would vanish very near the body profile (Note that [68] is nothing but $\psi_{\xi}(\xi)$. Nothing has been done in our study to aroid this difficulty; we have smoothed the solution by means of [62], [63] and [64]

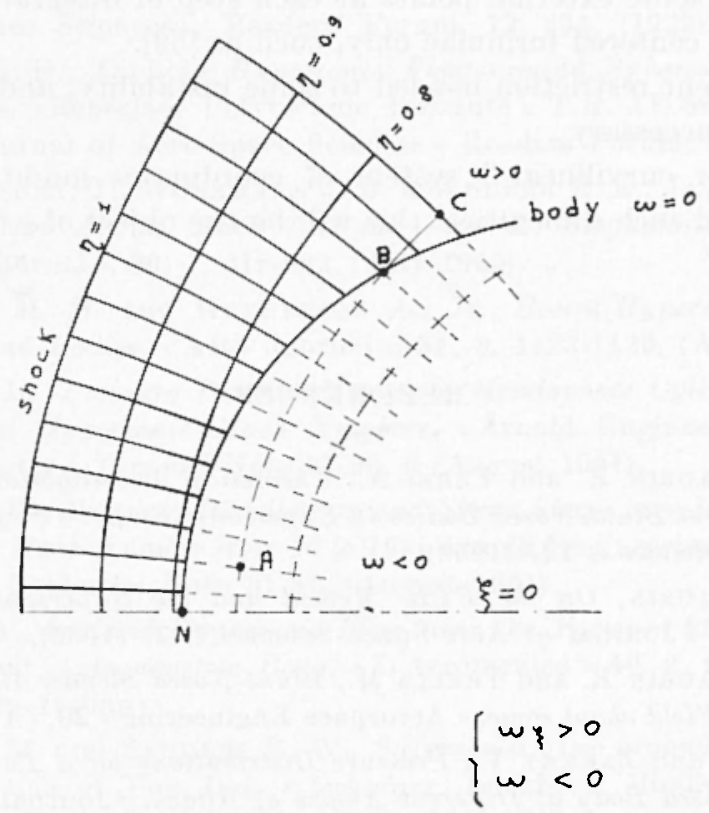

Fig. 20

till $y=0.122$ and then extrapolated the results to the body. We are studying the possibility of "leading" function $\xi_{x}\left(x_{1} ; y\right)$ ( $x_{t}$ fixed) to vanish at that particular value of $y$ where $v\left(x_{i} ; y\right)$ vanishes.

\section{VIII - CoNCluSIONS.}

A method of getting a " united approximation " of all the thermodynamic functions is given. The velocity components and the entropy are taken as unknowns, thus preparing the study of an axisymmetrical body at an angle of attack where neither the potential flow nor the stream function can be considered. Two "divisions by zero" are illustrated and it is shown that one of them certainly cannot occur in the subsonic field: it is then of no importance in connection with this study or similar ones. A way of avoiding the second division by zero is also indicated. 
A least squares method is used for differentiating the functions; a sinilar method is used three times for a smoothing process.

A wider field of Mollier diagram should be approximated in order 10 dispose of some external points at each step of integration to enable us to employ centered formulae only, such as [59].

The present restriction has led to some instability, and a smoothing process was neceessary.

A "polar curvilinear" system of coordinates might be useful in order to avoid such difficulties: this will be the object of a further study.

\section{REFERENCES}

(1) VAgito-Lauris R. and Ferri A., Theoretical Investigalion of the Flow Field about Blunt-Nosed Bodies in Supersonic Flight. "Journal of AeroSpace Sciences $n, 12$, (1958).

(2) Vaglio-Lauris, On the PLK Method and the Supersonic Blunt-Body Problem. "Journal of Aero-Space Sciences ", 2, (1962).

( $\left.{ }^{3}\right)$ Vaglio-Laurix R. and Trelia M., Blunt-Nosed Slender Bodies, A Study of Flow Field about some, "Aerospace Engineering" 20, (Aug. 1961).

(4) Fenki A. and Zakkax V., Pressure Distributions for a Two-Dimensional Blunt-Nosed Body at Different Angles of Attack. "Journal of Aero-Space Sciences ", 6, (1959).

(5) Hares W. D. and Pronstes R. F., Hypersonic Flow Theory. Academie Press, New York and London, 1959.

${ }^{6}$ ) Pronsters R. F., Shock Wave and Flow Field Development in Hypersonic Re-Entry. "ARS Journal ", 31, 2, 185-193, (Feb. 1961).

(') VAN DYKe II. D., The Supersonic Blunt-Body Problem - Review and Ertension. "Journal of Aero-Space Sciences" 8, (1.958).

(8) VAx Dyke M. D. and Gordox H. D., Supersonic Flow past a Family of Blunt Lrisymmetric Bodies. NASA T.R. R-1.

$\left({ }^{9}\right)$ Ripyard H. W., A Comparison of Blunt-Body Flow-Field Results. "Journal of Aero-Space Sciences" 27, 789-790, (Oct. 1960).

(10) Hamaker F. MI., Numerical Solution of the Flow of a Perfect Gas over a Circular Cylinder at Infinite Mach Number. NASA MEMO 2-25-59 A, Warch 1959.

(11) Axbrosio A. and Wortacax A., Stagnation Point Shock Detachment Distance for Hlow around Spheres and Cylinders. "ARS Journal", Rearlers' Forum, 32, 2, (Feb. 1962).

(12) VAX Hise V., Analytic Study of Induced Pressure on Long Bodies of Revolution with Tarying Nose Mluntness at Hypersonic Speed. NASA T.R. R 78, 1960. 
(13) Hansen C. F., Approximation for the Thermodynamic and Transport Properties of High-Temperature Air. NASA T.R. R 50, 1959, and NASA T.N. 4150, March 1958.

(14) Geiger R. E., On the Frozen Flow of a Dissociating Gas. "Journal of Aero-Space Sciences ", Readers' Forum, 12, 834, (1959).

(15) MaRtin E. D., Inviscid Hypersonic Flow around Spheres and Circular Cylinders. "Renselaer Polytechnic Institute" T.R. AE 5807, April 1958 and "Journal of Aero-Space Sciences" Readers Forum, 8, 529, (1959).

(16) Nagamatsu H. T., Workmans J. B. and Sineer R. E., Jr., Oblique Shock Relation for Air at Kach 7.8 and 7200 R. Stagnation Temperature. "ARS Journal ", 30, 7, 619-622, (July 1960).

(17) Bertray M. H. and Henderson A., Jr., Recent Hypersonic Studies of Wings and Bodies. "ARS Journal ", 31, 8, 1123-1139, (Aug. 1961).

(18) BAER A. L., Pressure Distributions on a Hemisphere Cylinder at Supersonic and Hypersonic Mach Numbers. "Arnold Engineering Development Center " Tecnical Note 61-96, 6 (August 1961).

(19) Lewis C. H., Pressure Distribution and Shock Shape over Blunted Slender Cones at Mach Number from 16 to 19. "Arnold Engineering Development Center "Technical Note 61-81, (August 1961).

${ }^{(20)}$ Muggia A., Inviscid Hypersonie Flow near the Forward Stagnation Point of a Blunt Axisymmetric Body. "LiAerotecnica" 40, 2, 112-118, (April 1960), (in Italian).

(21) Vixokur M. and SAxders R. W., Supersonic Flow: around Blunt Bodies, an extension of Tan Dyke's technique. Locklieed Missiles and Space Division, Tech. Rep. LAISD 48381. Jan. 1959.

(22) AVCo Manulacturing Corporation, Mollier Diagram for Equilibrium Air, AVCO Research Iuboratory January 1957, Everett, Massachusetts. 